\title{
The Chemistry of Lindo and Fages Colour Tests
}

\section{Francisco Sánchez-Viesca* and Reina Gómez}

Organic Chemistry Department, Faculty of Chemistry, National Autonomous University of Mexico, Mexico City (CDMX), Mexico; e-mail: franviesca@yahoo.com

* Corresponding author

\begin{abstract}
Lindo test for strychnine identification is based on the reaction of the alkaloid with potassium chlorate in the presence of sulphuric acid. Fages identified chlorates and bromates using strychnine in nitric acid. In fact, it is the same reaction but with different strong acid, and the product to be identified being interchanged. The reaction series occurring in these tests has not being described. We provide the electron flow from the alkaloid to the complex oxidation product resulting from the powerful oxidation properties of chloric acid.
\end{abstract}

\section{Introduction}

It is important ascertain what is happening in a spot test involving a substance with biological activity. Besides the theoretical interest of the subject, it reveals the chemical deportment of the compound. This result can be used to enhance, or on the contrary, deter the effect of the substance. This is related to Biochemistry, and in the case of strychnine, to toxicology and forensic medicine.

The color tests of Lindo and Fages involve the same reaction, differing in the mineral acid employed and in the object. That is, which of the substances that intervene is searched for. Since the reaction course is unknown, we provide the electron flow, step by step, from the alkaloid to the complex oxidation product whose structure was elucidated much later.

This communication is a follow up of our studies on reaction mechanisms, [1-5].

Received: November 10, 2020; Accepted: November 26, 2020

Keywords and phrases: 1,4-addition, chloryl cation, dismutation, epoxide formation, oxidations, strychnine identification.

Copyright () 2021 Francisco Sánchez-Viesca and Reina Gómez. This is an open access article distributed under the Creative Commons Attribution License, which permits unrestricted use, distribution, and reproduction in any medium, provided the original work is properly cited. 


\section{Antecedents}

The identification of strychnine by means of a colour test is due to Eugene Marchand $[6,7]$. He used lead dioxide and sulphuric acid with one percent nitric acid. A succession of colours is produced: violet-blue, purple and red. On prolonged standing it changes to orange and yellow.

This test prompted other chemists to try other oxidants. Thus, many cognate tests were produced and known by the names of the proposers. The sulphuric acid-dichromate test is described in Modern Methods of Plant Analysis [8]. In a book on Drug Identification, strychnine is recognized by the same test [9].

Following the mentioned trend, Lindo used potassium chlorate and sulphuric acid [10]. What is different in this test is the absence of the colour series which appears when other oxidizers are used. Only a red colour is observed.

Many years later Fages proposed the identification of chlorates and bromates using strychnine in nitric acid, although he mentioned that this reaction was well known for strychnine identification. The iodates and perchlorates do not give the colour reaction. His French communication [11, 12] was reviewed in England and in the United States $[13,14]$. The reaction product is the same as the obtained in the Lindo test since a red colour is produced, notwithstanding the different mineral acid employed. The use of nitric acid and chlorate for strychnine identification was recorded some years before Fages communication, the colour being reported as scarlet, [15].

Besides the dissimilar result compared to that observed in the other assays, Lindo test is interesting due to the special properties of chloric acid, the reactive species. It is a strong and unstable oxidizer, which in absence of a chemical reducer undergoes oxidoreduction reactions. The preparation of chlorine dioxide is based on the reaction of potassium chlorate with sulphuric acid. After formation of potassium sulphate and chloric acid, the last dismutates into chlorous acid and perchloric acid. Finally chlorine dioxide results from reaction of chloric acid with chlorous acid, [16].

\section{Discussion}

The strychnine molecule has the following groups: a lactam, a cyclic ether, a double bond, and a tertiary amine, Figure 1. 


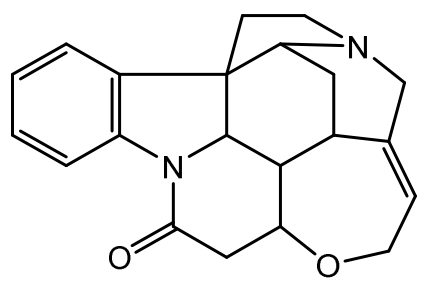

Figure 1. Strychnine structure.

The oxidation product isolated from potassium permanganate oxidation exhibits a carbonyl group vicinal to the tertiary nitrogen, that is, a new lactam group. There are also a ketone and a carboxylic acid. An uptake of four oxygen atoms has occurred, [17].

We provide the electron flow from the alkaloid to the oxidation product described, Figure 2. Since chloric acid is a powerful oxidant, it will accomplish the described oxidations to the final product. On the other hand, the functional groups obtained are in the higher oxidation state. So, there will be no further oxidation.

Chloric acid results from the reaction of potassium chlorate with sulphuric acid. Dehydration of chloric acid by protolysis affords the reaction species, chlorine dioxide cation (chloryl cation). This trigonal ion reacts with strychnine double bond, Figure 2, a. A transient carbonium ion is formed, $\mathbf{b}$, which is neutralized by proton elimination from the vicinal methylene group, enamine being formed, $\mathbf{c}$.

Chlorous acid is eliminated by protolysis and the double bond is reformed, now conjugated with an iminium ion, $\mathbf{d}$. This ion is neutralized by reaction with water, giving a carbinolamine, e. The hydroxyl group is oxidized to carbonyl after reaction with a chloryl cation, $\mathbf{f}$. Another molecule of chlorous acid is formed and a lactam results, $\mathbf{g}$.

1,4-Addition of chloric acid to the conjugated system yields an intermediate, $\mathbf{h}$, that is broken down by protolysis, with concomitant epoxide formation, i. This oxirane is opened in the acidic medium, $\mathbf{j}$, and a carbocation far to the carbonyl is formed, $\mathbf{k}$.

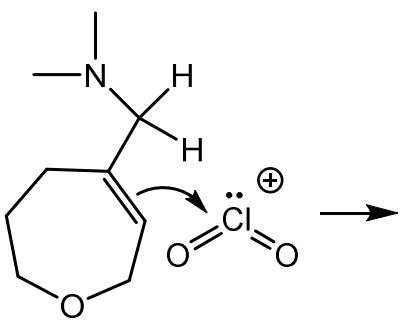

$\mathbf{a}$

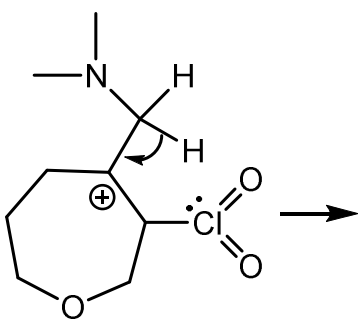

b

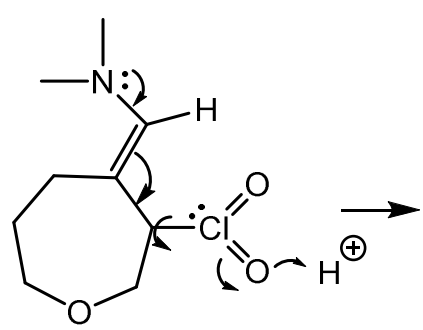

c 


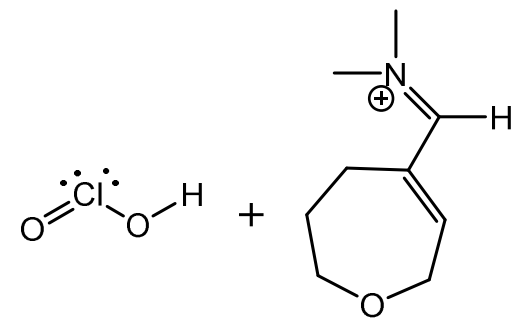

d

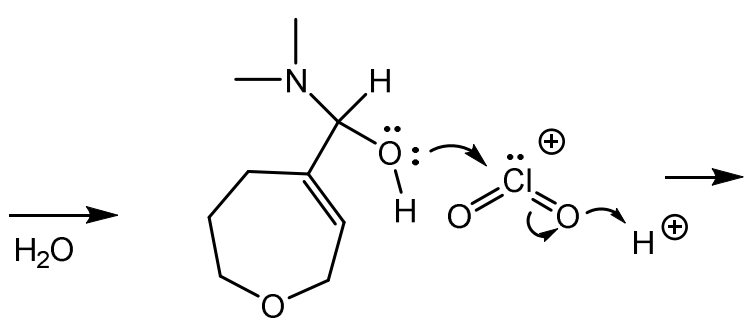

e

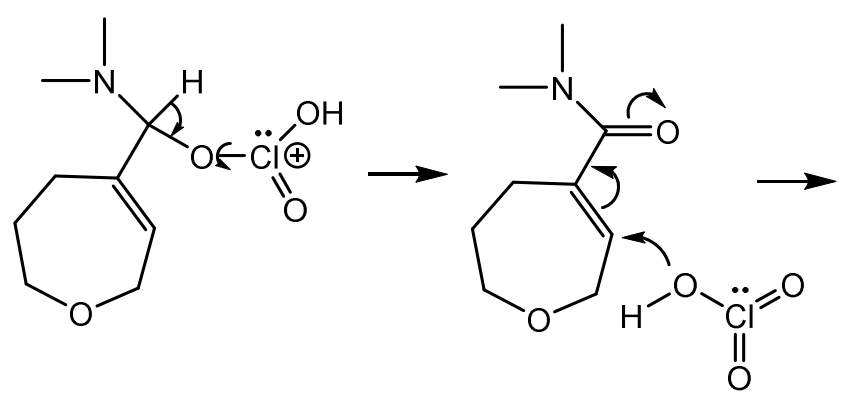

f

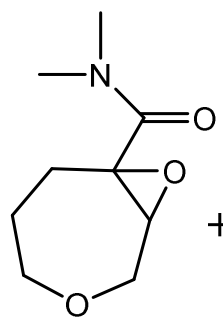

i

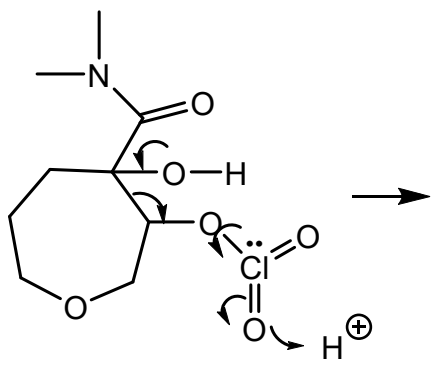

I g

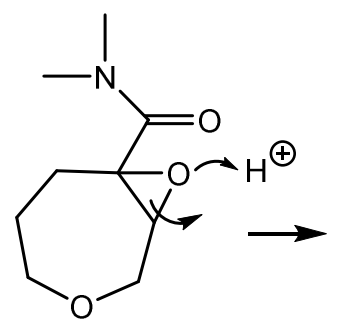

j

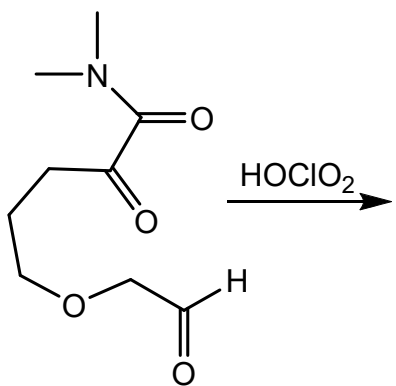

m

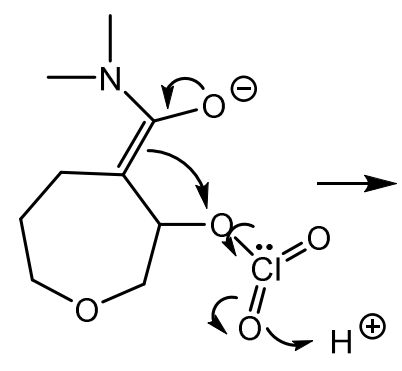

h

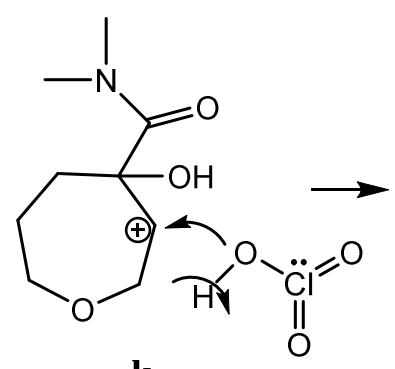

$\mathbf{k}$

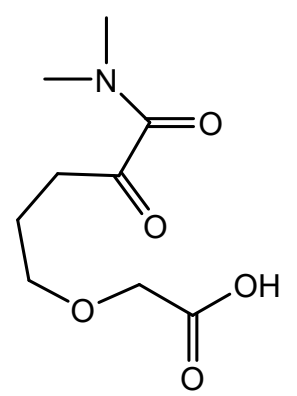

n

Figure 2. Sequence of strychnine reaction with potassium chlorate in acidic medium. 
Reaction of intermediate $\mathbf{k}$ with chloric acid, followed by protolysis, $\mathbf{l}$, gives chlorous acid and an aldehyde results via opening of the seven member ring, $\mathbf{m}$.

Finally, the aldehyde is oxidized to carboxylic acid, arriving to the complex oxidation product, $\mathbf{n}$.

The oxidation of the aldehyde occurs by protonation by means of chloric acid and the chlorate anion neutralizes the carbonium ion. Protolysis of the chlorate intermediate (addendum) yields chlorous acid and the $\mathrm{C}-\mathrm{H}$ group of the aldehyde is broken by a concerted mechanism.

The remarkable oxidation properties of chloric acid are observed when a mixture of sugar and potassium chlorate is ignited by addition of sulphuric acid, giving instant fire.

The first colours observed with other oxidants are missing in the Lindo test. This result is explained by the chemical deportment of chloric acid, the red colour being produced very fast (higher reaction rate).

The reduction product, chlorous acid, is unstable. Its instability is due to its tendency to a dismutation reaction to produce hypochlorous acid and chloric acid. This autooxidation/reduction occurs at ordinary temperature, $[18,19]$.

The growing acidity of the four oxy-acids of chlorine was correlated by Kossel with the increasing oxidation state of the chlorine, from hypochlorous to perchloric acid. The elimination of a proton gives an anion that stabilizes the central positive charge at the halogen, [20]. This is important since it helps explain the inorganic mechanisms that take place in the mentioned inorganic reactions.

The negative reaction with iodates and perchlorates mentioned by Fages can be explained by steric hindrance. The red colour, scarlet, observed in these tests is due to halochromism [21], proper of a substance in strong acidic medium (coloured salt formation).

\section{Conclusion}

We have traced the antecedents of both Lindo and Fages colour tests that involve strychnine and potassium chlorate. These assays are related notwithstanding the different mineral acid employed as solvent and catalyst.

Strychnine undergoes successive oxidations, but no reaction course is known. Thus, we provided the electron flow of the many steps that occur during the test. These steps 
are: attack to the double bond, carbinolamine formation, allylic oxidation, 1,4-addition, epoxide intermediate, two ring-openings, aldehyde formation and oxidation to carboxylic acid.

The reactive species are chloric acid and chloryl cation.

This way the reaction course from the alkaloid to the described final product has been cleared up.

Other items have been also discussed

\section{References}

[1] F. Sánchez-Viesca and R. Gómez, On the mechanism of uric acid oxidation with lead dioxide and with alkaline hydrogen peroxide, Indian J. Adv. Chem. Sci. 8(3) (2020), 7880 .

[2] F. Sánchez-Viesca and R. Gómez, The mechanism of nitric acid degradation of the Cglycoside Aloin to Aloe-emodin, Am. J. Chem. 10(1) (2020), 1-5.

[3] F. Sánchez-Viesca and R. Gómez, Formation mechanism of the colored compounds derived from Eserine (Physostigmine), World J. Org. Chem. 8(1) (2020), 1-4. https://doi.org/10.12691/wjoc-8-1-1

[4] F. Sánchez-Viesca and R. Gómez, On the mechanism of the Treumann test for theobromine, Int. J. Chem. Sci. 3(6) (2019), 46-49.

[5] F. Sánchez-Viesca and R. Gómez, The chemistry of the Folin test for uric acid, Am. $J$. Chem. 9(3) (2019), 91-94.

[6] E. Marchand, Sur un nouveau caráctère spécifique de la strychnine, Journal de Pharmacie et de Chimie, 3rd. series 4(1) (1843), 200-201.

[7] E. Marchand, New test for strychnine, Am. J. Pharm. 15 (1844), 300-301.

[8] K. Paech and M. B. Tracey, Modern Methods of Plant Analysis, Vol. 4, B. T. Cromwell, The Alkaloids, III, The detection of strychnine, 2, Colour Tests, p. 483, Berlin: Springer, 1980.

[9] C. A. Johnson, A. D. Th. Jones, Drug Identification, London: The Pharmaceutical Press, 1966, p.49.

[10] D. Lindo, Tests for strychnine, Assoc. Medical Journal 4(201) (1856), 966. https://doi.org/10.1136/bmj.s3-4.201.966

[11] J. Fages, Recherches des chlorates et des bromates au moyen de la strychnine, Bull. des Sciences Pharmacologiques 4 (1901), 15. 
[12] J. Fages, Recherche des chlorates et des bromates au moyen de la strychnine, Ann. Chim. Anal. Paris 5 (1900), 441-442.

[13] J. Fages, Detection of chlorates and bromates, The Pharmaceutical Journal (London) 12 (1901), 191.

[14] J. Fages, Chlorates and bromates, detection by means of strychnine, Proceedings of the American Pharmaceutical Association 49 (1901), 762.

[15] J. D. Mann, Forensic Medicine and Toxicology, London: Griffin, 1893, p. 548.

[16] A. Bruylants, J. C. Jungers and J. Verhulst, Química Mineral, Barcelona, Spain: Teide, 1965 , p. 101.

[17] R. W. Hoffmann, Classical Methods in Structure Elucidation of Natural Products, Zürich, Switzerland: Wiley-VHCA, 2018; 19: 193-197.

https://doi.org/10.1002/9783906390819

[18] E. Wiberg and A. F. Holleman, Inorganic Chemistry, San Diego, USA: Academic Press, 2001, p. 444.

[19] C.T. Rawcliffe and D. H. Rawson, Principles of Inorganic and Theoretical Chemistry, London: Heinemann, 1969, p. 310.

[20] F, D. de Körösy, An Approach to Chemistry, London: Pitman \& Sons, 1969, p. 192.

[21] M. Pesez and P. Poirier, Méthodes et Réactions de l'Analyse Organique, vol. 3, Paris: Masson, 1954, Halochromie, p. 227. 